\title{
Emotive Stimuli-triggered Participant-based Clustering Using a Novel Split-and-Merge Algorithm
}

\author{
Surabhi S Nath \\ Computer Science and Engineering \\ $3^{\text {rd }}$ B. Tech., IIIT Delhi \\ New Delhi, Delhi, India \\ surabhi16271@iiitd.ac.in
}

\author{
Dyutiman Mukhopadhyay \\ Experimental Psychology \\ University College London \\ London, UK \\ dyutimanm@gmail.com
}

\author{
Krishna P Miyapuram \\ Computer Science and Cognitive Science \\ IIT Gandhinagar \\ Gandhinagar, Gujarat, India \\ kprasad@iitgn.ac.in
}

\begin{abstract}
EEG signal analysis is a powerful technique to decode the activities of the human brain. Emotion detection among individuals using EEG is often reported to classify people based on emotions. We questioned this observation and hypothesized that different people respond differently to emotional stimuli and have an intrinsic predisposition to respond. We designed experiments to study the responses of participants to various emotional stimuli in order to compare participant-wise categorization to emotion-wise categorization of the data. The experiments were conducted on a homogeneous set of 20 participants by administering 9 short, one to two minute movie clips depicting different emotional content. The EEG signal data was recorded using the 128 channel high-density geodesic net. The data was filtered, segmented, converted to frequency domain and alpha, beta and theta ranges were extracted. Clustering was performed using a novel recursive-split and merge unsupervised algorithm. The data was analyzed through confusion matrices, plots and normalization techniques. It was found that the variation in emotive responses of a participant was significantly lower than the variation across participants. This resulted in more efficient participant-based clustering as compared to emotive stimuli-based clustering. We concluded that the emotive response is perhaps a signature of an individual with a characteristic pattern of EEG signals. Our findings on further experimentation will prove valuable for the progress of research in cognitive sciences, security and other related areas.
\end{abstract}

\section{CCS CONCEPTS}

- Theory of computation Unsupervised learning and clustering • Applied Computing Psychology • Hardware Neural systems/Digital signal processing • Human-centered computing $\sim$ Laboratory experiments

Permission to make digital or hard copies of all or part of this work for personal or classroom use is granted without fee provided that copies are not made or distributed for profit or commercial advantage and that copies bear this notice and the full citation on the first page. Copyrights for components of this work owned by others than ACM must be honored. Abstracting with credit is permitted. To copy otherwise, or republish, to post on servers or to redistribute to lists, requires prior specific permission and/or a fee. Request permissions from Permissions@acm.org. CoDS-COMAD '19, January 3-5, 2019, Kolkata, India

(C) 2019 Association for Computing Machinery. ACM ISBN 978-1-4503-62078/19/01 \$\$Psidoloi.org/10.1145/3297001.3297040

\section{KEYWORDS}

Clustering, EEG, Emotional Recognition, K-means, Brain Computer Interface

\section{INTRODUCTION AND RELATED WORK}

Electroencephalogram (EEG) is a simple, non-invasive and commonly used technique for studying the functioning of the human brain. EEG signals record electrical potentials and wave patterns generated by the complex network of neurons. EEG analysis is often performed for biomedical applications like epilepsy, autism etc. [1].

The detection of emotions using EEG has generated a considerable body of research over the years [reviewed in ref. 2]. Many of these works claim that emotions are similar and synchronized across people [3,4]. Another study claimed that human response to the environment is a result of their perception of the stimulus rather than the stimulus itself [5]. While event related synchronizations with respect to emotional stimuli have been observed, it has been remarked that EEG cannot differentiate between the various emotional states and can only reliably measure level of arousal [6]. Further, over a decade ago, it was stated that every individual has a unique EEG brain wave pattern [7] and more recently, a study confirmed the potential of EEG brain wave patterns as a means for unique identification of a person [8].

On the algorithmic data analysis, Steinley reviewed the evolution of K-means clustering and described the numerous formulations and algorithms used over the past fifty years [9]. A more recent paper discussed the agglomerative and divisive approach to Kmeans in detail [10]. An in-depth comparison of hierarchical and non-hierarchical clustering strategies was presented with the help of Monte Carlo simulations. It was verified that algorithms using correlation as the similarity measure led to more accurate results than those using Euclidean distances [11].

We believe that every individual is unique, embodied with different personality traits and responds differently to varying situations. We designed experiments to study the categorization of participants based on their response to emotive stimuli. The data was analyzed using a novel clustering algorithm and the results were simultaneously compared with the more established categorization of emotions using EEG recordings. This paper reports our findings. 


\section{MATERIALS AND METHODS}

\subsection{Data Acquisition}

\subsubsection{Participants}

Twenty participants, a mix of male and female candidates from M. Tech. and PhD programs at IIT Gandhinagar Neuroscience department aged around 22-26 years volunteered for the study in which they were required to watch short movie clips with emotional scenes from Bollywood movies. The study protocol was approved by Institutional Ethics Committee of IIT Gandhinagar.

\subsubsection{Design of Experiment}

The movie clips were of short duration approx between 2 and 3 minutes each. Each clip was chosen in order to portray a specific dominant emotion - the nine Rasas including beauty, laughter, sorrow, anger, courage, fear, disgust, surprise and peace [12]. A 10 second gap was included between successive movie clips. The total experiment session was nearly 30 minutes per candidate. A questionnaire was administered where all participants rated valence and arousal of the emotions on a scale of 9 for each clip.

\subsubsection{Data Collection Methodology}

Data was collected from 128 channel EGI (Philips) EEG system at $250 \mathrm{~Hz}$ sampling frequency.

\subsection{Preprocessing \& Data Extraction}

The short movie clips administered served as natural stimuli that elicit the inherent response from subjects. Further, since the stimuli were continuous and non-repeating, we chose not to resort to domain specific feature extraction and instead used the raw data for our analysis. The entire time signal was directly converted into frequency domain which enabled dimensionality reduction. This was done using Spectopo, an inbuilt function in EEGLAB which acquires the mean log spectrum of a set of data epochs at all channels as a bundle of traces, and obtains the relative topographic distribution of power at specified frequencies. The signals were segregated into theta $(5-8 \mathrm{~Hz})$, alpha $(9-13 \mathrm{~Hz})$ and beta $(14-30 \mathrm{~Hz})$ ranges. Since spectral intensities decrease with increasing frequencies, frequencies greater than $30 \mathrm{~Hz}$ were neglected. To reduce noise and dimension, the theta, alpha and beta ranges were down-sampled to one sample, which was represented by the median signal intensity value. The outer ring of electrodes near the eyes, nose, ears and face was ignored in order to collect data from the scalp region alone. The data was normalized using Eq. (1) in order to remove variability in signal intensity.

$\forall_{\text {row } i} \forall_{\text {column } \mathbf{j}} \quad \mathbf{X}(\mathbf{i}, \mathbf{j})=\mathbf{X}(\mathbf{i}, \mathbf{j})-\operatorname{median}(\mathbf{X}(\mathbf{i}))$

\subsection{Data Clustering}

The data matrix of $9 \times 20 \times 90$ (9 different types of emotional movie stimuli, 20 participants, 90 selected electrodes) were obtained each for theta, alpha and beta frequency ranges. This 3D matrix was reshaped into 180 x 90 2D matrices in two ways:

1) By concatenating person-wise

2) By concatenating emotive response-wise

We performed supervised classification on the dataset with train:test $=80: 20$ to group with respect to type of emotional stimuli. However, this led to low accuracy outputs (less than 20\%) on various classifiers like multiclass SVM, Discriminant Analysis, Naive Bayes, K Nearest Neighbours and Decision Tree.

We attempted unsupervised clustering for observing the natural similarities by analyzing the samples that cluster together without forcing labels on the data. We applied K-means, a popular, adaptable clustering technique that allows us to obtain the most appropriate distinguishing features in the data. Since our data indicated some outliers, we used K-medoids to disregard them by choosing median distance instead of the mean while assigning data elements to a cluster. We set the distance parameter to 'Spearman', which calculates the rank correlation according to Eq. 2. Correlation distances are sensitive to outliers.

\section{Spearman Correlation Coefficient}

$$
\rho=1-\frac{6 \sum d_{i}{ }^{2}}{n\left(n^{2}-1\right)}
$$

where $d$ is the difference between ranks and $n$ is the number of observations.

(2)

The K-medoids implementation on the data with $\mathrm{k}=9$ led to imbalanced clusters with about $50 \%$ of the data belonging to only two clusters. In order to tackle the imbalance of cluster population, we proposed a hierarchical K-medoids algorithm with recursive splitting with an upper limit on the cluster sizes (Figure 1).

\section{Figure 1: Illustration of recursive splitting}

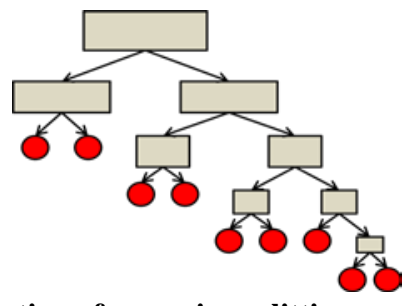

The splitting algorithm used was as follows (Figure 2):

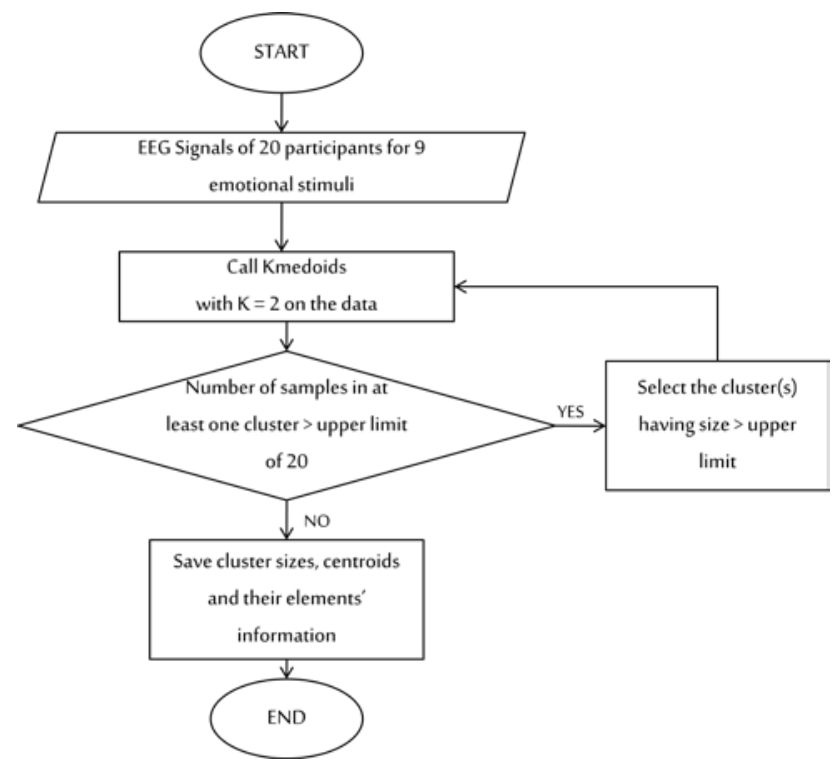

Figure 2: Flowchart for recursive splitting 
Use of the above algorithm resulted in the formation of several clusters, most containing very few samples. Thus along with fixing the upper limit, we also set a lower bound in a novel "splitand-merge" clustering algorithm. The merge strategy used a threshold value to reassign the clusters with sizes less than threshold into a different cluster having size greater than the threshold. The merge algorithm is shown below (Figure 3).

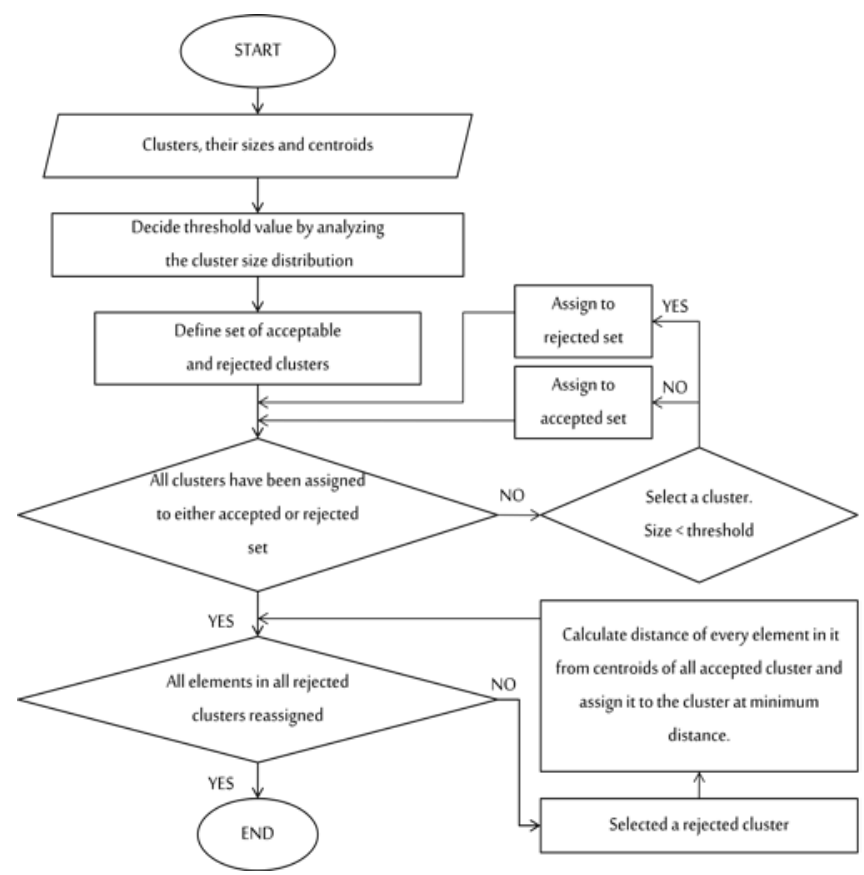

Figure 3: Flowchart for merging

\subsection{Methods of Data Analysis}

The clusters were then analyzed through topoplots, confusion matrices, F1 scores, and normalizations.

An array of size 180 was maintained where the $\mathrm{i}^{\text {th }}$ index stored the cluster number to which $i^{\text {th }}$ sample was assigned. The mode cluster number and its frequency was calculated for the following two cases:

1) The array is arranged in groups of 20, representing the 20 participants for each emotive stimulus. Nine mode values and corresponding frequencies were obtained. A confusion matrix was formed which displayed the distribution of the 20 participants in the clusters for all 9 emotive stimuli.

2) The array is arranged in groups of 9, representing the 9 emotive responses of each person. Twenty mode values and corresponding frequencies were obtained. Another confusion matrix was formed which displayed the distribution of the 9 emotive responses in the clusters for all 20 participants.

Mean F1-score was calculated for each confusion matrix as follows (Eq. (3)):

$$
\begin{gathered}
\text { F1score }=\frac{1}{\frac{1}{\text { precision }}+\frac{1}{\text { recall }}} \\
\text { Precision }=\frac{\text { True Positive }}{\text { True Positive+False Positive }} \\
\text { Recall }=\frac{\text { True Positive }}{\text { True Positive }+ \text { False Negative }}
\end{gathered}
$$

where value is considered true when cluster number matches the mode cluster number.

Net score was calculated by averaging the F1scores.

In addition, the mode person number and mode emotive response for every cluster was determined and stored.

Two other normalization techniques were used to fine-tune the results as follows:

1) For every electrode, the median signal value of the 20 participants for each emotive stimulus was subtracted from each of those 20 values.

2) Similarly, for every electrode, the median signal value of the 9 emotive responses of each participant was subtracted from each of those 9 values.

\section{RESULTS AND DISCUSSION}

The results are discussed under various heads as follows:

\subsection{Topoplots}

The distribution of brain activity in the cortex was observed through topoplots. The accepted cluster centroids (Figure 3) were represented and the highly excited regions were found in the outer circular area of the scalp, while the central region was found to be less active (Figure 4).

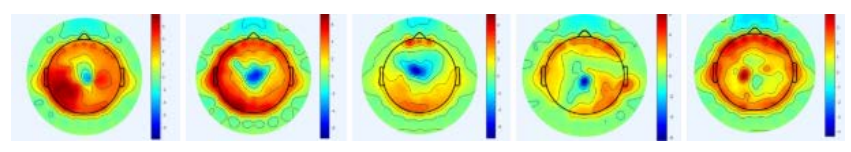

Figure 4: Sample topoplots of some of the cluster centroids

\subsection{Frequency of Mode Cluster}

The 9 frequencies of the mode cluster number obtained through emotive-stimulus-wise grouping were considerably lower than the ideal 20 (Figure 5a). On the other hand, high values near the ideal 9 were found for participant-wise grouping (Figure 5b).
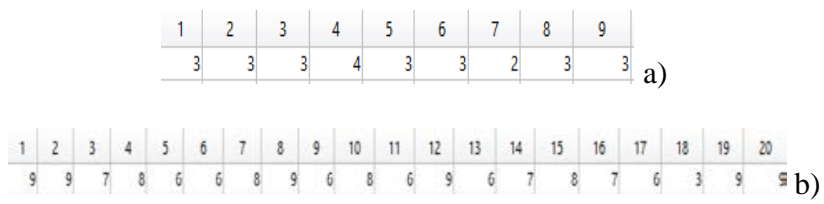

Figure 5: a) Frequency of the mode cluster number for each emotive stimulus b) Frequency of the mode cluster number for each participant 


\subsection{Confusion Matrix}

Ideally, the confusion matrix should have one high value and rest 0 indicating proper clustering. For emotive stimulus-wise clustering, the confusion matrix was mostly populated by small non-zero positive values with a mean score of 0.14 whereas the matrix for participant-wise clustering had mostly 0s interspersed with a few high values and a mean score of 0.78 . These observations reveal that participant-wise clustering clearly outperformed emotive stimulus-wise clustering.

In case of the participant-wise clustering, the mode cluster number for a participant matched the mode participant number in that cluster with 95\% accuracy on an average. On the other hand, in emotive stimulus-wise clustering, the mode cluster number for a stimulus matched the mode stimulus number in that cluster with only $20 \%$ on an average. This further strengthens our finding that application of participant-wise clustering is far superior to clustering using emotive stimuli in EEG-based signal analysis.

\subsection{Plots of EEG Signals}

The above results were validated using plots which showed that the variation of emotive responses of a participant was very small (Figure 6a) compared to the variation of the participants' response signals to a particular emotional stimulus which were considerably spread out (Figure 6b). Hence, we found that participant-based clustering was readily possible since each participant revealed a fixed wave pattern distinguishable from other participants. However, emotional stimuli-based clustering was not effective since no distinguishable pattern emerged across the various emotive stimuli.

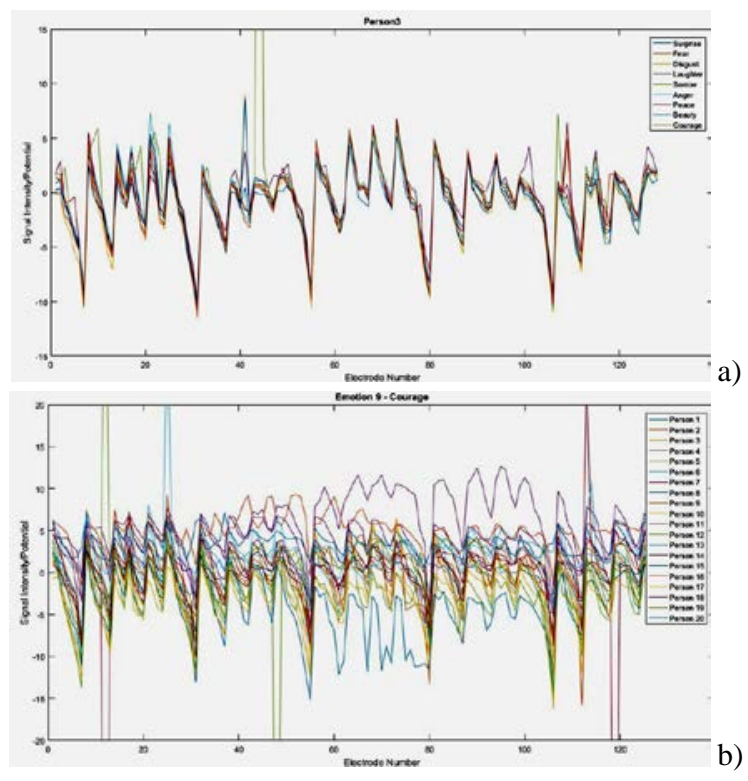

Figure 6: a) Sample graph of EEG waves of all emotional stimuli of one participant b) Sample graph of EEG waves of all participants for one emotional stimulus

\subsection{Normalization}

Finally, the normalization of the individual responses for the 9 emotive stimuli slightly improved emotive stimuli-wise clustering but reduced performance of participant-wise clustering since it reduced the cross participant differences. However, the normalization of the 20 participants' responses for each emotive stimulus did not significantly affect the already obtained results, thus confirming the validity of our findings.

\section{CONCLUSIONS}

The work demonstrated that the responses of participants for a given emotive stimuli was not similar despite selecting a homogeneous sample of participants for this experiment. We also observed that though a person responds differently to various emotional stimuli, the variation is significantly lower than variation across participants. This led to a better clustering based on participant as compared to clustering based on emotional stimuli. The emotive response is perhaps a signature of an individual with a characteristic EEG signal pattern. These findings can open new avenues for future research.

\section{ACKNOWLEDGMENTS}

SSN thanks the Summer Research Internship Programme (SRIP) 2018, for providing her a project opportunity at IIT Gandhinagar.

\section{REFERENCES}

[1] Tapan Gandhi, Bijay Ketan Panigrahi, Manvir Bhatia, Sneh Anand. 2010 Expert model for detection of epileptic activity in EEG signature. Expert $\begin{array}{llll}\text { Systems with 37(4), Applications. 3513-3520. } & \text { 37 }\end{array}$ DOI: 10.1016/j.eswa.2009.10.036

[2] Abeer Al- Nafjan et al. 2017. Review and Classification of Emotion Recognition Based on EEG Brain-Computer Interface System Research: A Systematic Review. Appl.Sci. 7, 1239. DOI:10.3390/app7121239

[3] Junichi Chikazoe, Daniel H Lee, Nikolaus Kriegeskorte and Adam K Anderson. 2014. Population coding of affect across stimuli, modalities and individuals. Nature Neuroscience. DOI: 10.1038/nn.3749.

[4] Cornell University. 2014. Study cracks how brain processes emotions. ScienceDaily. www.sciencedaily.com/releases/2014/07/140709135836.htm

[5] Tobias Brosch et al. 2009. The perception and categorisation of emotional stimuli: A review. DOI: 10.1080/02699930902975754

[6] Marni Y. V. Bekkedal, John Rossil III and Jaak Panksepp. 2011. Human brain EEG indices of emotion: Delineating responses to affective vocalizations by measuring frontal theta event-related synchronization. Neuroscience and $\begin{array}{lll}\text { Biobehaviorial } & \text { Reviews. } & \text { 35(9), }\end{array}$ DOI: 10.1016/j.neubiorev.2011.05.001

[7] M. Teplan. 2002. Fundamentals of EEG Measurement. Measurement Science Review. 2(2).

[8] Maria V. Ruiz-Blondet et al. 2016. CEREBRE: A Novel Method for Very High Accuracy Event-Related Potential Biometric Identification. IEEE Transactions on Information Forensics and Security. 11(7). DOI: 10.1109/TIFS.2016.2543524

[9] Douglas Steinley. 2010. K-means Clustering: A Half-century Synthesis. British Journal of Mathematical and Statistical Psychology. DOI: 10.1348/000711005X48266

[10] M. Venkat Reddy, M. Vivekananda and R U V N Satish. 2017. Divisive Heirarchical Clustering with K-means and Agglomerative Heirarchical Clustering. Int. J. Comp. Sci. Trends Technol., 5(5), 6-11.

[11] Dieter Scheibler and Wolfgang Schneider. 1985. Monte Carlo Tests of the Accuracy of Cluster Analysis Algorithms: A Comparison of Hierarchical and Nonhierarchical Methods. Multivariate Behavioral Research. 20(3), 283-304. DOI: $10.1207 / s 15327906 m b r 2003 \_4$

[12] R. Tripathi, D. Mukhopadhyay, C. K. Singh, K. P. Miyapuram, S. Jolad. 2018. Characterizing Functional Brain Networks and Emotional Centers based on Rasa Theory of Indian Aesthetics. arXiv:1809.05336v1. 